\title{
DEFICIENCIA INTRÍNSECA ESFINTERIANA. ¿QUÉ PAPEL JUEGA LA HIPERMOTILIDAD URETRAL EN SU MANEJO?
}

Intrinsic sphincter dysfunction. What is the role of urethrall hypermobillity in. management?

Alejandra Salazar-González, $M D^{1}$; Luis Guillermo Echavarría-Restrepo, $M D, M S c^{2}$

Recibido: noviembre 26/12 - Aceptado: febrero 18/14

\section{RESUIMEN}

Objetivo: reflexionar sobre la importancia del diagnóstico de la hipermotilidad uretral en el manejo quirúrgico de la disfunción intrínseca del esfínter uretral y la evaluación que se ha hecho de la hipermotilidad en los estudios que evalúan las diferentes técnicas para su corrección.

Conclusión: son pocos los estudios en pacientes con diagnóstico de deficiencia esfinteriana intrínseca que han evaluado la tasa de curación y porcentaje de complicaciones posoperatorias en pacientes llevadas cirugía con cabestrillos TVT y TOT teniendo en cuenta la presencia o no de hipermotilidad uretral.

Palabras clave: incontinencia urinaria de esfuerzo, uretra, urodinámica, cabestrillo suburetral.

1 Ginecoobstetra, Universidad Pontificia Bolivariana, Unidad MaternoInfantil, Clínica Universitaria Pontificia Bolivariana, Medellín, Colombia. alejasg@gmail.com

2 Profesor titular Ginecología y Obstetricia, Universidad Pontificia Bolivariana. Magíster en Salud Pública, Universidad de Antioquia. Magíster en Epidemiología, Universidad CES. Ginecoobstetra, coordinador de posgrado en Ginecología y Obstetricia, Universidad Pontificia Bolivariana, Medellín, Colombia. Líder científico Unidad Materno-Infantil, Clínica Universitaria Pontificia Bolivariana, Medellín, Colombia.

\section{ABSTRACT}

Objective: To reflect on the importance of diagnosing urethral hypermobility in the surgical management of intrinsic urethral sphincter dysfunction, and on the assessment of hypermobility carried out in the studies that have evaluated the different techniques used for addressing the problem.

Conclusion: Only a few studies in patients diagnosed with intrinsic sphincter dysfunction have evaluated cure rates and percentage of postoperative complications in patients taken to surgery with TVT and TOT slings, taking into account the presence or absence of urethral hypermobility.

Key words: Stress urinary incontinence, urethra, urodynamics, suburethral sling.

\section{INTRODUCCIÓN}

La incontinencia urinaria (IU) es una condición frecuente en las mujeres, con una prevalencia aproximada de $15 \%(1,2)$. Existen varios tipos de IU: incontinencia urinaria de urgencia, de esfuerzo y mixta. La incontinencia urinaria de esfuerzo (IUE) se define como la pérdida involuntaria de orina con el aumento de la presión abdominal (tos, estornudo, valsalva) (3), clasificada en 1988 por Blaivas y Olson con base en dos mecanismos anatómicos (4), el 
primero es el mecanismo de soporte o componente extrínseco, integrado por la vagina en su porción anterior, la fascia endopélvica, el arco tendinoso fascia-pelvis y el elevador del ano; su falla genera alteración del soporte del cuello vesical produciendo hipermovilidad uretral. El segundo mecanismo o componente intrínseco consiste en el sistema de cierre del esfínter uretral compuesto por los músculos estriado y liso uretral, además de elementos vasculares que contribuyen al cierre de la uretra en reposo; la alteración del mecanismo intrínseco genera deficiencia intrínseca del esfínter (DIE) (5); puede haber daño de los dos mecanismos descritos coexistiendo así una hipermotilidad uretral asociada a DIE (6). Desde nuestro punto de vista la variable que más influye en el éxito quirúrgico de las pacientes, independientemente del tipo de IUE, es la hipermotilidad uretral.

El objetivo del presente artículo es hacer una reflexión sobre la importancia de la evaluación de la motilidad uretral como elemento clave para tener en cuenta en el diagnóstico y el manejo quirúrgico de DIE, como también acerca de cuánto se ha tenido en cuenta la motilidad uretral en los estudios que evalúan las diferentes técnicas para su corrección.

\section{DIAGNÓSTICO DE DEFICIENCIA INTRÍNSECA DEL ESFÍNTER (DIE) Y LA HIPERMOTILIDAD URETRAL}

El concepto de DIE fue descrito por McGuire et al., en 1980 (6). Estos autores, mediante la realización de estudios urodinámicos, fluoroscopia y videourodinamia establecieron los criterios para la clasificación de la severidad de la IUE y DIE, estos son: la presión máxima de cierre uretral (PMCU), la presión de fuga abdominal.

La base fisiopatológica de la DIE está relacionada con el daño del mecanismo intrínseco uretral. Inicialmente se asoció a cirugías pélvicas o uretrales previas, denervación del piso pélvico, radioterapia y fibrosis, que se acompañaban de uretras fijas. El hipoestrogenismo también estaría asociado como consecuencia de la pérdida del tono basal de la uretra o pérdida del mecanismo de sello (7).

Para el diagnóstico de DIE la medida más utilizada hoy en urodinamia es la presión de fuga abdominal, la cual se diagnostica cuando dicha presión es $<60$ cm $\mathrm{H}_{2} \mathrm{O}$ (8-12). Por otra parte, Ghoniem propone clasificar la DIE en grados A, B y C (7) de acuerdo con la apertura del cuello vesical y la hipermotilidad uretral definidas por videofluorodinamia, y la presión de fuga abdominal definida por una PCUM $<10 \mathrm{~cm} \mathrm{H}_{2} \mathrm{O}$.

La DIE-A se refiere a la pérdida funcional del sello uretral en pacientes con cuello vesical cerrado en reposo e hipermotilidad uretral; DIE-B se refiere a una uretra con cuello vesical abierto en forma de pico e hipermotilidad con estrés, y la DIE-C es una uretra fija no móvil, con cuello vesical abierto e IU severa, que sugiere historia quirúrgica o radiación previa. Ellos proponen, en las pacientes con diagnóstico de DIE-A, DIE-B y DIE-C, iniciar tratamiento con terapia de inyección Bulking, si esta conducta falla y las pacientes presentan hipermotilidad uretral, es decir, las clasificadas en DIE-A y DIE-B, la opción es el manejo quirúrgico con cabestrillo suburetral. Cuando no hay hipermotilidad uretral (uretra fija) sugieren iniciar el manejo con la liberación de la uretra (uretrolisis) (11).

\section{HIPERMOTILIDAD Y EVALUACIÓN DEL TRATAMIENTO QUIRÚRGICO CON LOS CABESTRILLOS SUBURETRALES}

En los pocos estudios que tienen en cuenta la hipermotilidad uretral, en pacientes con DIE se ha evidenciado mayor riesgo de falla en aquellas pacientes que presentan uretras fijas y DIE, en comparación con pacientes con DIE e hipermotilidad uretral asociada (13).

En el 2008, Poza et al. publican un estudio que evalúa 254 mujeres con IUE llevadas a manejo quirúrgico con cabestrillo TOT; se tiene en cuenta la 
hipermotilidad uretral y la presencia de DIE (definida en su estudio con una PCUM $<20 \mathrm{~cm} \mathrm{H}_{2} \mathrm{O}$ ), con resultados más favorables en las pacientes que tenían solo hipermotilidad uretral previa a cirugía en comparación con las pacientes con uretras fijas. $\mathrm{Al}$ año de seguimiento, en su cohorte de estudio encontró un porcentaje de falla del 10,6\% en el grupo de hipermotilidad uretral en comparación con el 20,7\% de falla en el grupo de uretra fija (13).

De otra parte, hay varios estudios que avalan el uso preferencial del TVT sobre el TOT para el manejo de la DIE los cuales, al comparar las dos técnicas, reportan tasas de curación superiores y necesidad menor de reintervención quirúrgica a los tres años de seguimiento (14-17). Sin embargo, estos estudios no informan sobre la presencia o no de hipermotilidad uretral y no definen parámetros similares de estandarización en el diagnóstico de DIE.

\section{CONCLUSIÓN}

Actualmente, la literatura muestra superioridad del cabestrillo TVT sobre TOT para el manejo de DIE, pero la mayoría de los estudios no tienen en cuenta la hipermotilidad uretral. Otra dificultad metodológica en estos estudios es la falta de estandarización de los parámetros urodinámicos para el diagnóstico de DIE. Se requieren estudios en pacientes con diagnóstico de deficiencia esfinteriana intrínseca que evalúen la tasa de curación en pacientes llevadas a cirugía con cabestrillos suburetrales TVT y TOT, teniendo en cuenta la presencia concomitante o no de hipermotilidad uretral.

\section{REFERENCIAS}

1. Nygaard I, Barber MD, Burgio KL, Kenton K, Meikle S, Schaffer J, et al. Prevalence of symptomatic pelvic floor disorders in US women. JAMA. 2008;300:1311-6.

2. Álvaro R, Araco F, Gravante G, Sorge R, Overton J, Vellone E, et al. Epidemiological aspects of urinary incontinence in a female population of an Italian region. Int Urogynecol J. 2010;21:873-83.

3. Haylen BT, de Ridder D, Freeman RM, Swift $\mathrm{SE}$, Berghmans B, Lee J, et al. An International
Urogynecological Association (IUGA)/International Continence Society (ICS) joint report on the terminology for female pelvic floor dysfunction. Neurourol Urodyn. 2010;29:4-20.

4. Blaivas JG, Olsson CA. Stress incontinence: classification and surgical approach. J Urol. 1988;139:727-31.

5. McGuire EJ. Diagnosis and treatment of intrinsic sphincter deficiency. Int J Urol. 1995;2(Suppl 1):7-10.

6. McGuire EJ, Lytton B, Kohorn EI, Pepe V. The value of urodynamic testing in stress urinary incontinence. J Urol. 1980;124:256-8.

7. McGuire EJ, Woodside JR. Diagnostic advantages of fluoroscopic monitoring during urodynamic evaluation. J Urol. 1981;125:830-4.

8. McGuire EJ. Urodynamic findings in patients after failure of stress incontinence operations. Prog Clin Biol Res. 1981;78:351-60.

9. McGuire EJ, Fitzpatrick CC, Wan J, Bloom D, Sanvordenker J, Ritchey M, et al. Clinical assessment of urethral sphincter function. J Urol. 1993;150:1452-4.

10. Hosker G. Is it possible to diagnose intrinsic sphincter deficiency in women? Curr Opin Urol. 2009;19:342-6.

11. Ghoniem GM, Elgamasy AN, Elsergany R, Kapoor DS. Grades of intrinsic sphincteric deficiency (ISD) associated with female stress urinary incontinence. Int Urogynecol J Pelvic Floor Dysfunct. 2002;13:99-105.

12. Petros PE, Ulmsten UI. The combined intravaginal sling and tuck operation. An ambulatory procedure for cure of stress and urge incontinence. Acta Obstet Gynecol Scand Suppl. 1990;153:53-9.

13. Poza JL, Pla F, Sabadell J, Sánchez-Iglesias JL, Martínez-Gómez X, Xercavins J. Trans-obturator suburethral tape for female stress incontinence: a cohort of 254 women with 1-year to 2-year follow-up. Acta Obstet Gynecol Scand. 2008;87:232-9.

14. Latthe PM. Review of transobturator and retropubic tape procedures for stress urinary incontinence. Curr Opin Obstet Gynecol. 2008;20:331-6.

15. Torres G, Lujan M, Martín C, García A, Rodríguez N, Berenguer A. [TVT and TOT for surgical correction of female stress urinary incontinence. Comparison between techniques]. Arch Esp Urol. 2008;61:861-5. 
16. Stavros C, Ioannis V, Vasileios SI, Gkotsi AC, Georgios S, Papathanasiou A, et al. Comparison of TVT, TVT-O/ TOT and mini slings for the treatment of female stress urinary incontinence: 30 months follow up in 531 patients. Arch Ital Urol Androl. 2012;84:129-36.
17. Schierlitz L, Dwyer PL, Rosamilia A, Murray C, Thomas E, De Souza A, et al. Effectiveness of tensionfree vaginal tape compared with transobturator tape in women with stress urinary incontinence and intrinsic sphincter deficiency: a randomized controlled trial. Obstet Gynecol. 2008;112:1253-61.

\section{Conflicto de intereses: ninguno declarado.}

